\title{
Power System Voltage Stability Classification Using Interior Point Method Based Support Vector Machine (IPMSVM)
}

\author{
Hwachang Song ${ }^{1}$, Rodel D. Dosano ${ }^{2}$ and Byongjun Lee ${ }^{3}$ \\ ${ }^{1}$ Department of Electrical Engineering, Seoul Nat'l University of Technology, Seoul 139-743, Korea \\ 2 School of Electronic and Information Eng., Kunsan Nat'l University, Kunsan 573-701, Korea \\ ${ }^{3}$ School of Electrical Engineering, Korea University, Seoul 136-701, Korea
}

\begin{abstract}
This paper presents a methodology for the classification of power system voltage stability, the trajectory of which to instability is monotonic, using an interior point method based support vector machine (IPMSVM). The SVM based voltage stability classifier can provide real-time stability identification only using the local measurement data, without the topological information conventionally used.
\end{abstract}

Key Words: classification, interiorpointmethod, localmeasurement, voltagestabilityidentification, supportvector machine

\section{Introduction}

With the advent of deregulated energy markets and the growing desire to fully utilize existing transmissions and infrastructures, power system stability is becoming complex and critical. This economical pressure on an electrical market forces the operation of power systems and components to the limits of capacity, and system conditions can be more exposed to instability due to greater uncertainty in day to day system operations and the increase in the number of critical components. Thus, the evolution of electric system operation schemes toward the deregulation necessitates more reliable security monitoring.

The integrated SCADA/EMS system is crucial for the current power system operation, and its capability has greatly improved during recent years. Obviously, however, the SCADA/EMS system has a difficulty to capture dynamic responses after severe disturbances occur. To compensate for this difficulty, phasor measurement units (PMU) was devised and proposed in the power engineering field [1], which fulfills the requirement of real-time monitoring for the view of power system dynamic responses. Time series data from the fast measurement devices can provide the insight of system dynamics to the operators. Potentially, in addition, they can provide system stability identification through adequate data manipulation. Using synchronized PMU data with time tags from GPS (Global Positioning Systems), concepts of wide area control/protection systems (WACS/WAPS) were proposed [2,

Manuscript received Mar. 17, 2009; revised Jun. 15, 2009.

Corresponding Author : hcsong@snut.ac.kr (Hwachang Song)

This work was supported by the Korea Ministry of Knowledge Economy through the EIRC program at Korea University.
3], which have a complete dynamic view through the systems and provide an appropriate control action in an emergency state.

This paper proposes a new methodology for real-time local voltage stability classification with a support vector machine (SVM). SVM is said to provide an excellent performance in binary classification in a multi-dimensional feature space [4-6]. The approach of this paper directly uses time-series data of phasor measurement on system trajectories in insecure states. From a set of data samples in a moving window, in this paper, a feature vector is generated with a procedure for letting the vector contain relative information from the pre-fault condition, and from the generated feature vectors as the learning samples, this paper adopts an interior point method based support vector machine (IPMSVM) to determine the optimal separating hyperplane, which will be then used for unseen feature vectors. This paper also depicts the solution technique of IPMSVM, basically using the interior point method for nonlinear programming problems in [7], and explains a numerical example with Jeju island power system [8] to show that the classifier can provide very effective signals for long-term voltage stability identification.

\section{IPM based support vector machine}

This section explains the formulation and solution technique for the IPMSVM. SVM, to find an optimal hyper- 
plane in the hyperthesis space separting the feature vectors into two classes, can be formulated as a quadratic programming (QP) problem with linear constriants. When applying interior point methods to QP problems, the total iteration number does not severely depend on the increased problem size [9].

\subsection{Formulation}

This section presents the SVM formulation and the solution technique used in the paper. Suppose we have $N$ training data points $\left\{\left(x_{1}, y_{1}\right),\left(x_{2}, y_{2}\right), \ldots \ldots,\left(x_{N}, y_{N}\right)\right\}$ where $x_{i} \in R^{d}$ and $y_{i} \in\{ \pm 1\}$, where $x_{i}$ denotes the i-th feature vector and $y_{i}$ is the corresponding class (positive or negative class). For non-separable cases, the optimization formulation of SVM, to find out the hyperplane $(w \cdot x+b=0)$ maximizing the margin between the two classes, can be described as follows:

$$
\begin{array}{ll}
\min & \frac{1}{2} w^{T} w+C \sum_{i=1}^{N} \xi_{i} \\
\text { s.t. } & y_{i}\left(w \cdot x_{i}+b\right)+\xi_{i} \geq 1, \quad 1 \leq i \leq N \\
& \xi_{i} \geq 0, \quad 1 \leq i \leq N
\end{array}
$$

where $\xi_{i}$ is the slack variable for the i-th sample; $C$ is the weighting factor for penalizing $\xi_{i}$ s in the objective function. The first term of the objective function is to maximize the margin between the two classes. [5]:

The dual problem of (1) can be formulated as follows

$$
\begin{array}{ll}
\max & \sum_{i=1}^{N} \alpha_{i}-\frac{1}{2} \sum_{i=1}^{N} \sum_{j=1}^{N} \alpha_{i} \alpha_{j} y_{i} y_{j} x_{i} \cdot x_{j} \\
\text { s.t. } & 0 \leq \alpha_{i} \leq C, \quad 1 \leq i \leq N \\
& \sum_{i=1}^{N} \alpha_{i} y_{i}=0
\end{array}
$$

where $\alpha_{i}$ is the Lagrangian multiplier of the $i$-th inequality constraint in (1). Instead of the primal problem, the dual problem is usaully used for SVM solutions, because its applicability to nonlinear kernels is better. For a nonlinear SVM classifier, a nonlinear kernel, $K\left(x_{i}, x_{j}\right)$, needs to be substituted for $x_{i} \cdot x_{j}$ in (2). Support vectors by definition are those feature vectors that lie on the binding constraints and hence have positive Lagrangian multipliers, so each class can have several support vectors and they ultimately determine the optimal hyperplane.

After obtaining the optimal solution of (1), the classification of the output classes with an input vector $x$ can be determined by:

$$
\begin{aligned}
f(x) & =\operatorname{sgn}(w \cdot x+b) \\
w & =\sum_{i=1}^{N} \alpha_{i} y_{i} x_{i}
\end{aligned}
$$

From (3), it can be known that only those constraints corresponding to the support vectors could affect the normal vector calculation. This relation can be obtained from the optimality condition of the Lagrangian function.

\subsection{Solution Technique}

For implementation of SVM, in this paper, an interior point method (IPM) [7] is applied as the solution technique. For the dual problem of (2), after introducing slack variables $s_{L}$ and $s_{U}$ for converting the inequality constraints into equility ones and applying log barrier penalty functions for them, Lagrangian function can be obtained as follows:

$$
\begin{aligned}
& L=f(\alpha)-z^{T}\left(\alpha-s_{L}\right)-w^{T}\left(\alpha+s_{U}-C\right)-\lambda y^{T} \alpha \\
& \quad-\mu \sum_{i=1}^{N}\left(\ln s_{L i}+\ln s_{U i}\right) \\
& f(\alpha)=-\sum_{i=1}^{N} \alpha_{i}+\frac{1}{2} \sum_{i=1}^{N} \sum_{j=1}^{N} \alpha_{i} \alpha_{j} y_{i} y_{j} x_{i} \cdot x_{j}
\end{aligned}
$$

where $z$ and $w$ are Lagrangian multipliers for the converted lower and upper inequality constraints; $\lambda$ is a scalar Lagrangian multiplier for the equality constraint; $\mu$ denotes barrier parameter.

The KKT first-order necessary optimality condition of the Lagrangian function can be derived as follows:

$$
\begin{aligned}
& L_{\alpha} \equiv \nabla f(\alpha)-z-w-\lambda y=0 \\
& L_{\lambda} \equiv y^{T} \alpha=0 \\
& L_{z} \equiv \alpha-s_{L}=0 \\
& L_{w} \equiv\left(\alpha+s_{U}-C\right) \\
& L_{s_{L}} \equiv Z e-\mu S_{L}^{-1} e=0 \\
& L_{s_{U}} \equiv W e+\mu S_{U}^{-1} e=0
\end{aligned}
$$

where $S_{L}$ and $S_{U}$ are diagonal matrices whose diagonal elements are the elements of $s_{L}$ and $s_{U}$, respectively; $Z$ and $W$ are diagonal matrices whose diagonal elements are the elements of $z$ and $w$, respectively; $e$ is the vector whose all elements are 1. For easy manipulation of the KKT firstorder condition, the last two equations in (5) are changed into the following equations.

$$
\begin{aligned}
& \underline{L}_{s_{L}} \equiv S_{L} Z e-\mu e=0 \\
& \underline{L}_{S_{U}} \equiv S_{U} W e+\mu e=0
\end{aligned}
$$

From a given initial guess for the variables in (4), CityplaceNewton method is applied to find a solution satisfying (5). For this purpose, this paper uses the reduced correction equations as follows:

$$
\begin{aligned}
& {\left[\begin{array}{cc}
H & y \\
y^{T} & 0
\end{array}\right]\left[\begin{array}{c}
\Delta \alpha \\
\Delta \lambda
\end{array}\right]=-\left[\begin{array}{c}
R(\cdot) \\
y^{T} \alpha
\end{array}\right]} \\
& H(\cdot)=-\nabla^{2} f(\alpha)+S_{U}^{-1} W-S_{L}^{-1} Z \\
& R(\cdot)=-\nabla f(\alpha)+\lambda y+\mu\left(S_{L}^{-1} e-S_{U}^{-1} e\right)
\end{aligned}
$$

Other variable correction vectors are calculated with the following equations:

$$
\begin{aligned}
& \Delta s_{L}=\Delta \alpha+L_{z}^{o} \\
& \Delta s_{U}=-\Delta \alpha-L_{w}^{o} \\
& \Delta z=-S_{L}^{-1} Z\left(\Delta \alpha+L_{z}^{o}\right)+S_{L}^{-1} \underline{L}_{s_{L}}^{o} \\
& \Delta w=S_{U}^{-1} W\left(\Delta \alpha+L_{w}^{o}\right)-S_{U}^{-1} \underline{L}_{s_{U}}^{o}
\end{aligned}
$$

where $L_{z}^{o}, L_{w}^{o}, \underline{L}_{s_{L}}^{o}$ and $\underline{L}_{s_{U}}^{o}$ are vectors with intermediate numerical values for $L_{z}, L_{w}, \underline{L}_{s_{L}}$ and $\underline{L}_{s_{U}}$, respectively. 
After determining primal and dual step lengths not to linearly violate the inequality constraints and leave the room for the variables in the next iteration, primal and dual varaibles are updated with the correction vectors.

The procedure of IPMSVM for the optimal solution can be outlined as follows:

Step 1: Initialize the primal and dual variables, and set $k$ to 1 .

Step 2: Compute the complementary gap, $G_{C}=z^{T} s_{L}-$ $w^{T} s_{L}$, and the residual of the equality constraints of (2), $y^{T} \alpha$. If $G_{C}<\varepsilon_{1}$ and $y^{T} \alpha<\varepsilon_{2}$, then stop; otherwise, go to Step 3.

Step 3: Set the barrier parameter, $\mu$, as follows:

$$
\mu=c \frac{G_{C}}{2 n_{C}}
$$

where $n_{C}$ is the number of inequality constraints $(=2 N)$, and $c(0<c<1)$ is the centering parameter.

Step 4: Construct the linear system of (7) and solve the correction vector for $\left(\alpha, s_{L}, s_{U} ; \lambda, z, w\right)$.

Step 5: Determine the primal and dual step length, $\left(t_{P}, t_{D}\right)$, for the next solution not to violate any inequality constraints linearly.

Step 6: Update the primal and dual variables using the correction vector and $\left(t_{P}, t_{D}\right)$ as follows:

$$
\begin{aligned}
& {\left[\begin{array}{c}
\alpha^{(k+1)} \\
s_{L}^{(k+1)} \\
s_{U}^{(k+1)}
\end{array}\right]=\left[\begin{array}{c}
\alpha^{(k)} \\
s_{L}^{(k)} \\
s_{U}^{(k)}
\end{array}\right]+t_{P}\left[\begin{array}{c}
\Delta \alpha \\
\Delta s_{L} \\
\Delta s_{U}
\end{array}\right]} \\
& {\left[\begin{array}{c}
\lambda^{(k+1)} \\
z^{(k+1)} \\
w^{(k+1)}
\end{array}\right]=\left[\begin{array}{c}
\lambda^{(k)} \\
z^{(k)} \\
w^{(k)}
\end{array}\right]+t_{D}\left[\begin{array}{c}
\Delta \lambda \\
\Delta z \\
\Delta w
\end{array}\right]}
\end{aligned}
$$

Step 7: $k \leftarrow k+1$, and go to Step 2 .

In (9), $c$ is usually set to 0.1 , and then $G_{C}$ in the next iteration expects to be reduced ten times. Also $\mu$ gets lower as iteration goes by, and closer to a very small value (below $10^{-8}$ around the solution. The main computational burden stems from the calculation of the correction vector of the reduced system as in (7), the dimension of which is $N+1$. Because the system matrix of (7) is compact, it seems to be not possible to apply sparse techniques. However, the total iteration number, when applying IPM, one of polynomial time algorithms, tends not to change as the system size increases, and the computational time is proportional to the total iteration number. In this paper IPMSVM has been developed in Matlab.

\section{Feature vector generation for voltage stability classification}

In the literature, one can find several references that apply artificial intelligence technologies for the estimation of active power margin, which are also based on SCADA/EMS solution. Different from these approaches, this paper proposes a new methodology that directly uses time-series data from local measurements. The basic idea of the methodology is that time-trajectories for stable cases and those for unstable cases can be differentiated.

In this paper, we assume that the parameters, locally monitored by PMU devices, are active power and bus voltage $(P$ and $V)$ at the pilot substation. The two parameters are usually used for the conventional voltage stability analysis [10-12]. The sampling frequency needs to be fast enough to capture system responses of long-term dynamics. A high sampling rate can contain the system response from short-term and long-term dynamics, but it increases the dimension of the feature space.

To convert time-series data into feature vectors, the concept of moving window is used. A window contains a certain number of samples, and the window slides along the time axis. The data points in a moving window are the basis for classification of voltage stability. A window at a specific time includes $M-1$ previous data points and a current data point, where $M$ is the size of the window. The dimension of the feature space is $2 M$, because each point has values of $P$ and $V$. At the beginning of classification, the classification output is provided after $M$ samplngs. In this paper, a preconditioning of the input data is performed for generation of feature vectors. Assuming that $P$ and $V$ values at the pre-faulted condition is stored, the preconditioning is done using the following formula:

$$
\begin{aligned}
& \underline{P}_{k}=\left(P_{o}-P_{k}\right) / P_{o} \\
& \underline{V}_{k}=\left(V_{o}-V_{k}\right) / V_{o}
\end{aligned}
$$

where the subscripts $o$ and $k$ represent the initial (pre-fault) and the $k$-th sample. The normalized parameters, $\underline{P}_{k}$ and $\underline{V}_{k}$, are more differentiable by classification algorithms, because they include relative information from the initial values.

For classifying a continuous sequence of generated feature vectors, several algorithms [12] can be employed. In this paper, IPMSVM is adopted as the classifier. SVM is powerful for binary classification in a multi-dimensional feature space and constructs an optimal decision hypothesis by holding the empirical risk fixed and minimizing VC confidence [4-6]. 


\section{Numerial results}

This section reports the results applying the SVM based voltage stability classifier to '05 Jeju island system in the KEPCO (Korea Electric Power Corporation). The total load of the system is 490.9 [MW] and $30.9 \%$ of the load is supplied from the main system through two HVDC lines [8]. The HVDC lines has the isochronous control which actually regulates the system frequency of the island system after disturbances by increase or decrease in the injected current through them. Thus, in cases where a large portion of generation in the island system is tripped off, a quick increase in active power injection is followed due to the isochronous control. Then, unless reactive power is sufficiently and quickly supported for the system to maintain loadability for the load restoration level, the system may experience voltage collapse.

This paper assumes that a local measurement device such as PMU is equipped at a substation to capture timeseries data for $P$ and $V$. The pilot bus for monitoring is 'Shin JEJU'. To obtain time series data, in this paper, TSAT program [14] is applied, and in the time-domain simulation, all the loads are converted by the ULTC based load restoration model and the HVDC controls are modeled with the user defined control (UDC) blocks in TSAT. During simulation, the network-side voltage and active power injection to the substation are stored for application of the proposed classifier.

In this study, diverse scenarios are simulated, to obtain stable and unstable data as learning samples, including outages of lines and local generators and increase in local loads for enough data sets. In preparation of data sets, the main idea in selection of major disturbances is to have the classifier acquire diverse and significant information in the learning process. Expertise in voltage stability is needed in determination of disturbance sets as a part of classifier modeling. Most data obtained in time-domain simulation were stable, so this results in much stable data and less for unstable data. The prepared learning samples, consequently, are more biased in positive than negative class and hence this might cause difficulties in further classification of unseen data.

In this study, 480 training data are prepared totally; 115 data of them are unstable, and used to verify the capability of the voltage stability classifier. All unstable training data with the equal number of randomly selected stable data were first adopted to train the classifier. Then, additional 30 data sets which are randomly chosen from the remaining stable class are incorporated into the previous learning samples and this procedure is repeatedly done until no unused training data are left. In this way, the characteristics of the SVM classifier are evaluated using different number of training data and comparative studies with other learning algorithms are performed.

The test data used in this study are generated from a typical scenario where the system is initially can be considered stable after applying the contingency but turns into unstable. That is, a time-series is created applying the scenario, including 128 stable and 35 unstable data points, and then several training data according to a moving window are prepared. The scenario is taken apart from other scenarios employed for the training data.

Using IPMSVM, developed in this paper, with linear and Gaussian kernel function, the training procedure was performed. For penalty parameter of SVM, $C$, various values are applied; $C$ for the linear kernel function ranges from 105000 and for Gaussian kernel function from 2000 to above. Selecting $C$ in SVM modeling is such an important part as choosing the kernel function and the number of training data. Since the number of unstable data for training is not so much as that of stable data in this study, it is expected that SVM solutions are affected by settings of C. In the authors' experiences with the classifier, solutions with linear kernel are very dependent on the number of training data, while those with Gaussian kernel are closely related to both the number of training data and $C$ parameter.

The parameters for training are $P$ and $V$ and both of them contribute in the detection of voltage stability classification. Since the voltage profiles of the test system are well-maintained, active power load is more sensitive to the system disturbances compared to the voltage magnitude in this study. These facts could be found in some test scenarios where the system is unstable but does not experience very low voltage profile. In Fig. 1 and 2, time-series data for $P$ and $V$ are illustrated, generated by a voltage unstable scenario. From the figures, it can be known that before the system becomes unstable, the system tries to recover voltage profile by ULTC actions but becomes more stressed by the load restoration action.

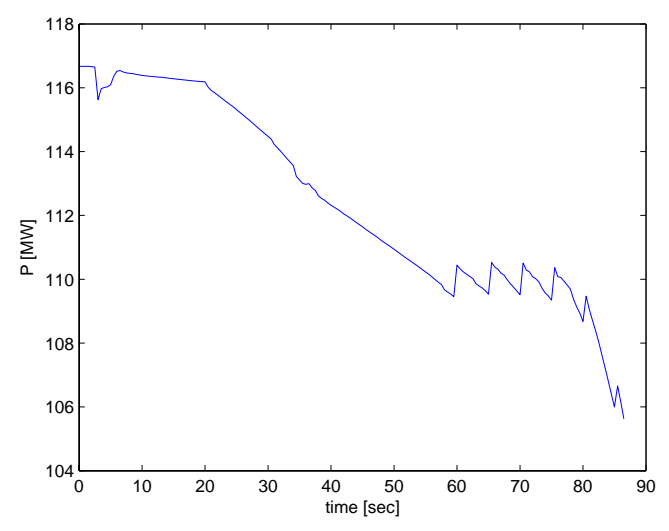

Fig. 1 Time trajectory of $P$ for the performance test 


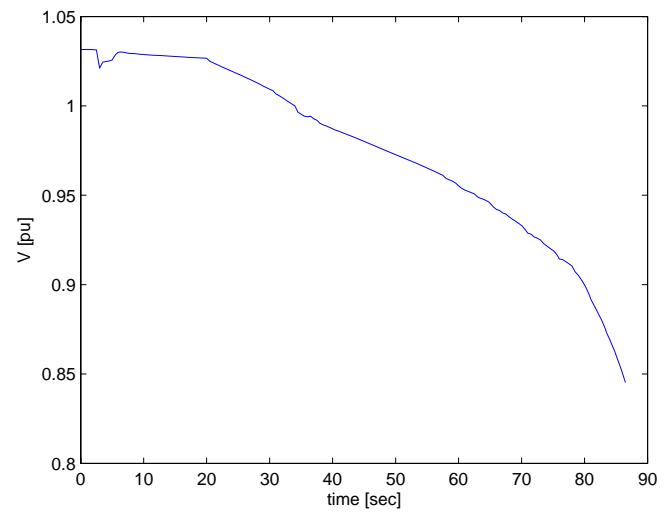

Fig. 2 Time trajectory of $V$ for the performance test

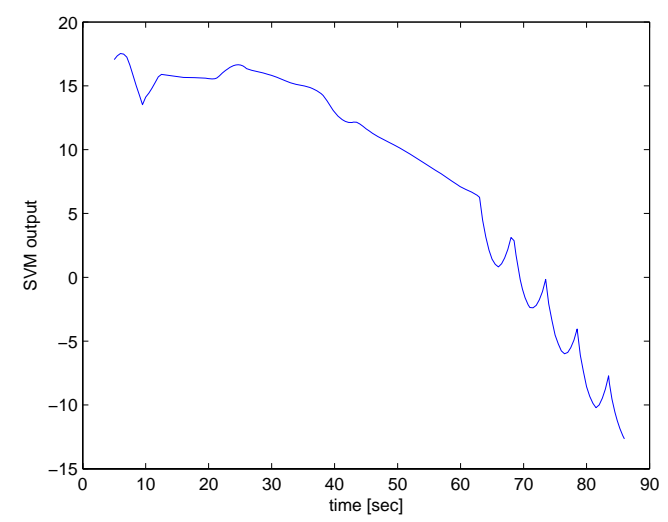

Fig. 3 SVM output for the voltage unstable case

Fig. 3 shows the SVM output for the test data. As the system starts to respond after the contingency, the SVM output changes noticeably. From the figure, one can roughly say that the larger the magnitude of SVM output is in positive, the more stable the system is. In the authors' experience, also, the severity of the disturbance on local voltage stability can be viewed from the SVM output; of stable cases, the more severe the disturbance is, the closer the SVM output is to the reference axis. Thus, SVM output can be used as an auxiliary signal because it is capable of tracking the direction of system responses in terms of voltage stability.

To observe the responses of the SVM output when control actions are applied, an adequate control of load shedding to save the system for the unstable case was conducted. The timing of load shedding action is one of important factors in determination of control strategies. In the case, if enough load shedding is applied before the magnitude SVM classifier output approaches the reference axis, the system could be back to a stable trajectory. The corrective control, as shown in Fig. 4, keeps the SVM output positive. The SVM output jumps rapidly to a much higher value immediately after applying the load shedding and then gets down and settles around a positive steady value.

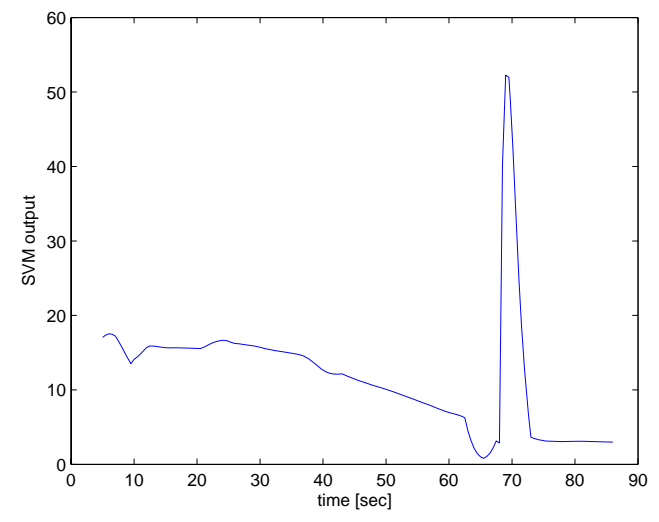

Fig. 4 SVM output when applying load shedding control for stabilization

A comparison study is done on the performance of IPMSVM with Multi-Layered Perceptrons (MLP) models. In this study, IPMSVM with linear (IPMSVM1) and Gaussian kernel (IPMSVM2) functions, Multi-Layered Perceptrons models (MLP1 and MLP2) with 5 and 10 hidden layers. For the classifiers, the same training and testing data sets are applied. In Table 1, detection and false dismissal rate are shown for each classifier when the number of training data is 480 . Detection rate, $R_{D}$, is the ratio of the stable test data that have been correctly classifier and false dismissal rate is $1-R_{D}$. For this configuration, IPMSVM2 have a best performance in terms of classification; the maximum detection rate that can be achieved using MLPs in this study is 0.9632 , but that using IPMSVM2 is 1. In Fig. 5 , detection rates of the classifiers with 300 and more training samples are illustrated. On the early stage of the graph, an important set of data from the boundaries of the positive class have not yet been included. The detection rate of IPMSVM2 gets improved after adding the appropriate positive class in the training data.

Table 1. Performance comparison with other classifiers

\begin{tabular}{|c|c|c|c|}
\hline & $\begin{array}{c}\text { Detection } \\
\text { rate }\end{array}$ & $\begin{array}{c}\text { False dismissal } \\
\text { rate }\end{array}$ & $\begin{array}{c}\text { number of } \\
\text { traning data }\end{array}$ \\
\hline MLP1 $(\mathrm{a}=5)$ & 0.9632 & 0.0368 & 480 \\
\hline MLP2 $(\mathrm{a}=10)$ & 0.9632 & 0.0368 & 480 \\
\hline IPMSVM1 & 0.9387 & 0.0613 & 480 \\
\hline IPMSVM2 & 1.0000 & 0.0000 & 480 \\
\hline
\end{tabular}

In the voltage stability classification, the ease of tuning the classifier and the consistency of results with unbalance training data are important factors for accurate detection. In selection of classifier, the compensation of data over-fitting, which is implicit in the nature of the problem, should be considered as one of major priorities. For power system application, most of the data available are for stable cases, so the generalization ability of SVM with limited resources for training fits this type of classification. 


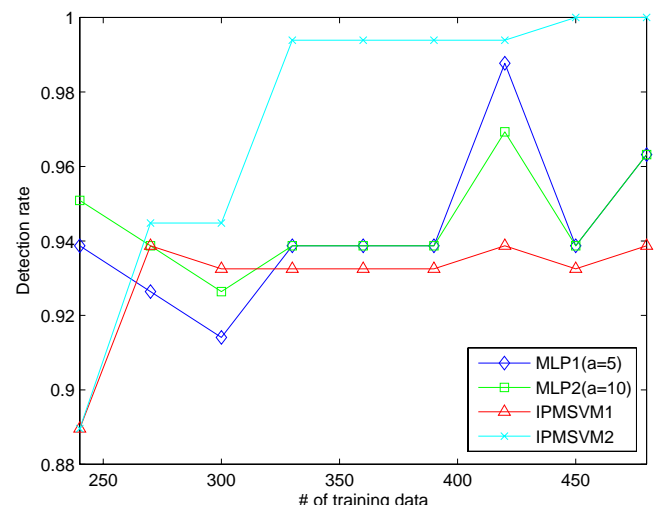

Fig. 5 Detectio $n$ rates of the classifiers by \# of training data

\section{Conclusion}

This paper presents a new methodology for real-time power system voltage stability classification using the support vector machine based on an interior point method. When applying IPM, the total number of iteration is not seriously affected by the increase in the system size, which depends on the number of samples for SVM. For feature vector generation, the classifier directly uses sets of time series data, which can be obtained by local measurement on system responses, with the simple manipulation to increase the relative information. The test results show that the classifier can offer an effective signal for long-term voltage stability identification based on real-time local measurements.

\section{References}

[1] R. O.Burnett Jr. and M. M. Butts, "Power system applications for phasor measurement units," IEEE Computer Applications in Power, vol. 7, no. 1, pp. 8-13, 1994.

[2] C. Rehtanz and J. Bertsch, "A new wide area protection system," Proc. of 2001 IEEE Power Tech Conference, vol. 4, 2001.

[3] C. W. Taylor, D. C. Erickson, K. E. Martin, R. E. Wilson, and V. Venkatasubramanian, "WACS - Wide area stability voltage control system: R and D and online demonstration," Proc. of IEEE, vol. 93, no. 5, pp. 892906, 2005.

[4] V. Vapnik, Statistical learning theory, John Wiley \& Sons, New York, 1998.

[5] N. Cristianini and J. Shawe-Taylor, An introduction to support vector machines and other kernel-based learning methods, Cambridge University Press, New York, 2000.

[6] C. J. C. Burges, A tutorial on support vector machines for pattern recognition, Online:www.umiacs. umd.edu/users/joseph/support-vector-machines4.pdf.

[7] S. Mebrotra, "On the implementation of a primal-dual interior point method," SIAM Journal on Optimization, vol. 2, no. 4, pp. 575-601, 1992.

[8] B.-M. Yang, C.-K. Kim, G.-J. Jung, and Y.-H. Moon, "Verification of hybrid real time HVDC simulator in Cheju-Haenam HVDC system," Journal of Electrical Engineering \& Technology, vol. 1, no. 1, pp. 23-27, 2006.

[9] J. H. Wright, Primal-dual interior-point methods, SIAM, Philadelphia, 1997.

[10] P. Kundur, Power system stability and control, McGraw-Hill, New York, 1994.

[11] C. Taylor, Power system voltage stability, McGrawHill, New York, 1994.

[12] T. Van Cutsem and C. Vournas, Voltage stability of electric power systems, Kluwer Academic Publisher, Boston, 1998.

[13] D. Michie, D. J. Spiegelhalter and C. C. Taylor (eds.), Machine learning, neural and statistical classification, Online: http:// www.amsta.leeds.ac.uk/ charles/statlog/whole.pdf.

[14] Powertech Labs, TSAT user manual, Powertech Labs Inc., 2007.

\section{Hwachang Song}

Assistant professor of Seoul Nat'1 Univ. of Technology Research Area: Nonlinear optimization, power system stability and control, and system modeling

E-mail : hcsong@snut.ac.kr

\section{Rodel D. Dosano}

Ph.D. student of Kunsan Nat'l University

Research Area: Power system control and topology theory application

E-mail : rodel_dosano@kunsan.ac.kr

\section{Byongjun Lee}

Professor of Korea University

Research Area: Power system blackout and wide area control and protection

E-mail : leeb@korea.ac.kr 